\title{
Acquisition of zero-delay matching in pigeons
}

\author{
NICHOLAS F. SKINNER \\ King's College, Lniversity of Westem Ontario \\ London. Canada V6A 2113
}

The present report describes a procedure for generating efficient zero-delay matching in pigeons. Introducing $\mathrm{a}$ third $\mathrm{CO}$ to disrupt the position preference characteristic of preacquisiton performance under two-choice matching conditions was followed almost immediately by a steady increase in correct responding to near errorless levels.

In an extensive review of their research with the complex discriminated operant. in which matching-to-sample (MTS) is defined as a conditional procedure whereby a standard or sample stimulus (ST) determines which of a number of comparison stimuli (COs) is correct, Cumming \& Berryman (1965) reported that, in pigeons. predictable. but dissimilar, response patterns are generated by simultaneous and zero-delay MTS paradigms. Simultaneous two-choice matching performance does not substantially exceed chance levels during the first few sessions, which are characterized by a strong position preference. Concomitant with the breakdown of the position preference. correct matching develops rapidly to near errorless levels after approximately 1.000 trials. By contrast, the "exceedingly ragged" and protracted nature of zero-delay acquisition functions led Cumming \& Berryman (1965) to conclude that although "... the simultaneous presence of the ST and $\mathrm{CO}^{\circ} \mathrm{s}$ is not required for learning ... the zero-delay case does represent an exceptionally difficult problem [p. 309]."

Following from the observation by Cumming \& Berryman (1965) that position preferences, while not heavy, "...increased for each bird prior to the acquisition of [zero delay] matching [p. 309]." the present study tested the following hypothesis: If. as in simultaneous matching. acquisition occurs contiguously with the breakdown of positional responding (Cumming \& Berryman. 1965), then zero-delay matching acquisition may be facilitated by the disruption of positional responding through the introduction of an additional $\mathrm{CO}$. in that a position preference in three-choice matching would produce reinforcement on only one-third of the trials rather than $50 \%$ reinforcement in the two-choice condition.

\section{METHOD}

The Ss were four naive 12-month-old pigeons (Ectopistes migratorius: P1, P2, P3, and P4) maintained at $85 \%$ of their free-feeding weights. A standard pigeon chamber was equipped with a response panel containing: (1) a transparent sample key: (2) transparent choice keys centered in a horizontal row below the sample key and housed in interchangeable units of one to eight keys: and (3) a food hopper located directly below the choice keys and illuminited with white light when mixed grain reinforcement was available. Sample and choice keys were illuminated by rear projection, with different combinations of Ilford gelatin filters mounted in slides. Blue, green, yellow, and red (Ilford color filters, Nos. 602, 604, 606, and 608, respectively) were the hues used throughout the experiment, with the composition and order of presentation randomized over trials. Experimental contingencies were controlled automatically by circuitry located in an adjacent room and masked by white noise.

During shaping, completion of a five-peck fixed-ratio requirement (FR 5) on the sample key produced immediate cessation of illumination of the sample key and simultaneous illumination of the choice key, with the hue terminated on the sample key. Reinforcement (4-sec access to mixed grain) was contingent upon a response on the choice key. There were 10 sessions, consisting of 60 trials per session ( 15 trials per hue).

With two-choice acquisition training, fulfillment of the FR 5 led to the illumination of each of two choice keys with a different hue. A peck on the choice key of hue identical to that on the extinguished sample key was defined as a correct response and earned Ss reinforcement. This was followed immediately by cessation of all chamber lighting for a 20 -sec blackout (BO), during which responding was ineffective. An incorrect response immediately initiated a $\mathrm{BO}$.

Positional responding was well established in all Ss after 15 sessions of two-choice training: consequently, for experimental Ss, three-choice matching was instituted during Session 16, and remained in effect for Sessions 16-25 for P4 (after which two-choice matching was reinstated for five sessions) and Sessions 16-30 for P3. 1 Control Ss (P1 and P2) received 30 sessions of two-choice matching.

During acquisition rraining, with order of presentation randomized, each choice key was designated correct on an equal number of trials. Each hue appeared an equal number of times on the sample key. and an equal number of times on both the correct choice key and incorrect choice key(s). In three-choice matching, the COs consisted of one identical in hue to the ST, plus two of the remaining three nonmatching hues. This provided a total of 72 possible $\mathrm{CO}$ combinations. Since there were 120 trials in each session. it was necessary to randomize the order of presentation of $\mathrm{CO}$ combinations over three sessions (rather than one, as was possible in the two-choice condition), i.e., over three sessions $(3 \times 120=360$ trials $)$, each $\mathrm{CO}$ combination appeared on five trials ( $5 \times 72 \doteq 360$ trials). The first and last 10 trials were not included in the data analyses.

\section{RESULTS AND DISCUSSION}

Zero-delay acquisition data are summarized in Tables 1-3. The development of matching under two-choice conditions was slow and irregular. Ss initially exhibited a slight position preference and an erratic increase in correct responding to a level marginally above chance. This pattern was gradually replaced by a consistent decline in the number of correct responses and a marked increase in the index of positional responding to a level averaging $60 \%$ over all $S$ s by Session 15. Correct responding for control Ss (Table i) continued to approximate chance during the remainder of the experiment. 
Table 1

Two-Choice Acquisition Data for Control Ss (P1 and P2)*

\begin{tabular}{|c|c|c|c|c|c|}
\hline Session & $\begin{array}{l}\text { Position } \\
\text { Prefe- } \\
\text { rence } \\
(c)\end{array}$ & $\begin{array}{l}\text { Number } \\
\text { of Correct } \\
\text { Matches } \\
\text { (Out of } \\
100)\end{array}$ & Session & $\begin{array}{c}\text { Position } \\
\text { Prefe- } \\
\text { rence } \\
(c)\end{array}$ & $\begin{array}{c}\text { Number } \\
\text { of Correct } \\
\text { Matches } \\
\text { (Out of } \\
100)\end{array}$ \\
\hline 1 & 15 & 45 & 16 & 55 & 52 \\
\hline 2 & 22 & 52 & 17 & 58 & 52 \\
\hline 3 & 13 & 49 & 18 & 57 & 54 \\
\hline 4 & 21 & 59 & 19 & 63 & 50 \\
\hline 5 & 19 & 62 & 20 & 60 & 54 \\
\hline 6 & 22 & 62 & 21 & 59 & 54 \\
\hline 7 & 17 & 64 & 22 & 58 & 53 \\
\hline 8 & 26 & 64 & 23 & 57 & 54 \\
\hline 9 & 37 & 65 & 24 & 57 & 54 \\
\hline 10 & 39 & 63 & 25 & 56 & 55 \\
\hline 11 & 41 & 62 & 26 & 56 & 58 \\
\hline 12 & 44 & 59 & 27 & 55 & 57 \\
\hline 13 & 45 & 57 & 28 & 54 & 56 \\
\hline 14 & 54 & 55 & 29 & 55 & 58 \\
\hline 15 & 59 & 52 & 30 & 54 & 57 \\
\hline
\end{tabular}

* Because of the highly similar acquisition functions of $P 1$ and $P$ 2. mean levels of position preference and correct responding are presented in Table 1 .

In contrast, introduction of the three-choice

Table 2

Two-Choice (Sessions 1-15) and Three-Choice (Sessions 16-30) Acquisition Data for Experimental S P3

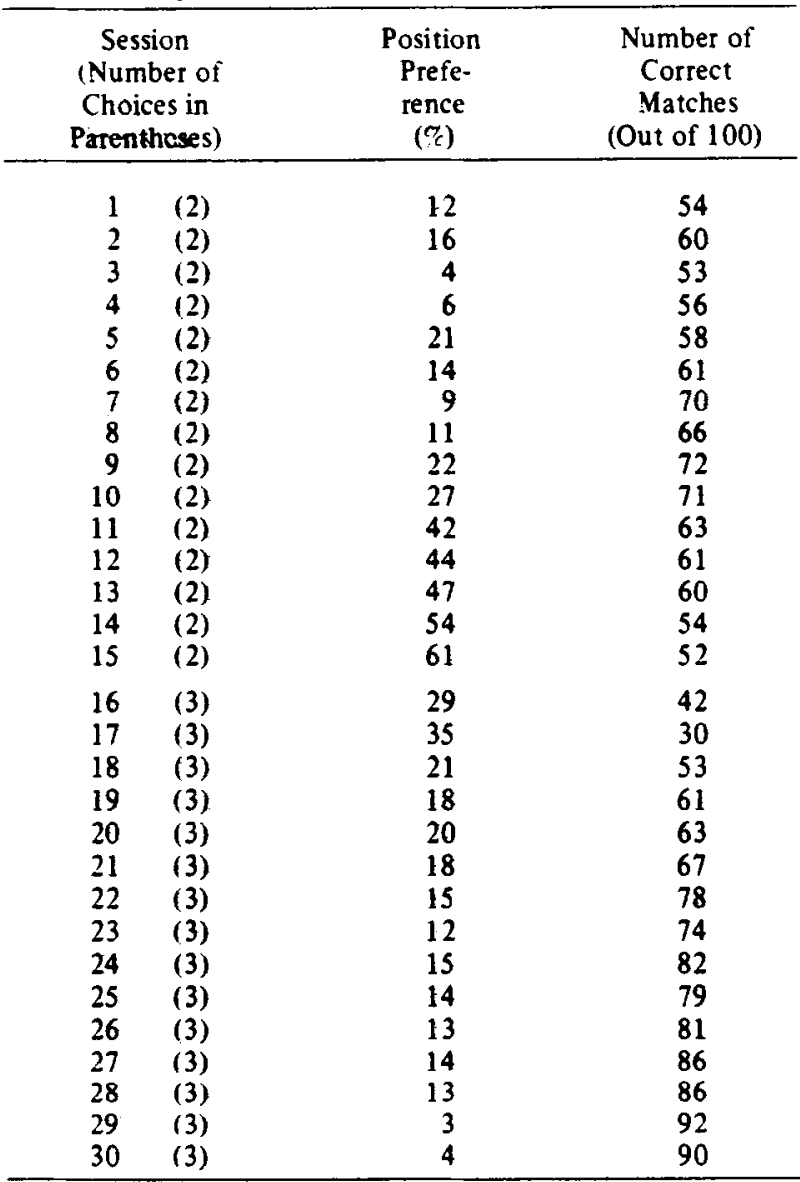

condition for experimental Ss (Tables 2 and 3 ) during Session 16 was followed by an almost uninterrupted decline in positional responding, and, after a further decrease in the number of correct matches for only one (P4) and two (P3) sessions, a pronounced rise in correct responding to near errorless levels. The reinstatement of two-choice matching for P4 in Sessions 26-30 led to the rapid establishment of correct response performance higher than that recorded at any point during prior exposure (Sessions 1-15).

The results suggest somewhat paradoxically that making the zero-delay task more difficult facilitates acquisition. Without further study, ${ }^{2}$ it cannot be stated categorically that this circumstance followed from the disruptive effects of the third $\mathrm{CO}$ on positional responding; but dramatic three-choice acquisition was obtained with the methods employed. Given the difficulties encountered by Cumming \& Berryman (1965), and in recognition of the adaptive importance of this form of discrimination learning (e.g., it requires recall rather than the simple recognition necessary for simultaneous matching), these preliminary findings ${ }^{3}$ may provide assistance and impetus for further research using the zero-delay MTS paradigm.

Table 3

Two-Choice (Sessions 1-15, 26-30) and Three-Choice (Sessions 16-25) Acquisition Data for Experimental S P4

\begin{tabular}{ccc} 
Session & Position & Number of \\
(Number of & Prefe- & Correct \\
Choices in & rence & Matches \\
Parentheses) & $(c)$ & $($ Out of 100) \\
\hline
\end{tabular}

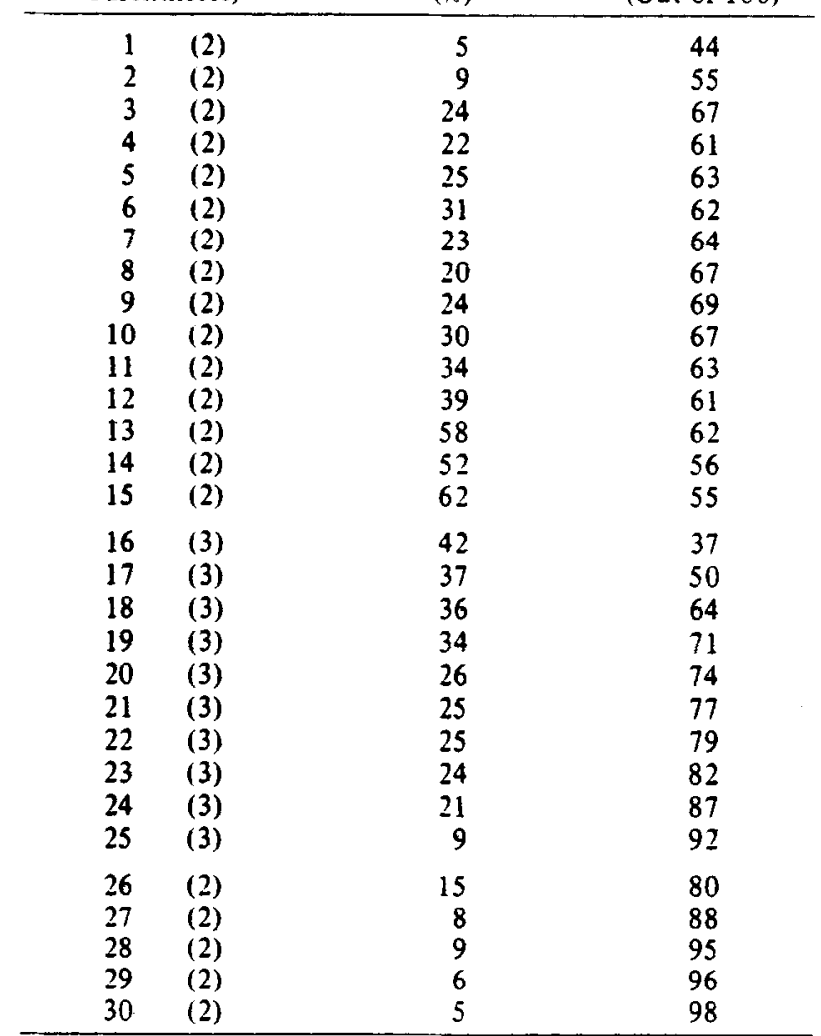




\section{REFERENCES}

Cumming, W. W., \& Berryman, R. The complex discriminated operant: Studies of matching-to-sample and related problems. In D. I. Mostofsky (Ed.), Stimulus generalization. Stanford: Stanford University Press, 1965. Pp. 284-330.

\section{NOTES}

1. Following the procedure of Cumming and Berryman (1965), for the two- and three-choice matching conditions, respectively, positional responding was computed so that if a particular choice key was chosen on one-half (or one-third) of the trials there would be no position preference; if it was chosen on every trial, there would be $100 \%$ preference.

2. For example, three and four choices might be used, with performance when choices are introduced after 15 sessions compared with acquisition in other Ss placed on three- and four-choice regimens from the outset.

3. The data reported here are taken from an investigation of the effects of multiple stimuli on choice reaction time in pigeons.

(Received for publication July 16,1973 ; revision received August 24, 1973.) 